\title{
Neural Network Parameter Adjustment for Control Rules in Flight Control System
}

\author{
Jing Zhou ,Jun Tao \\ School of Mathematics and Computer Science \\ Jianghan University, \\ Wuhan, Hubei, P.R.China
}

\begin{abstract}
To deal with the problem of parameter adjustment in flight control system, it is designed that parameter adjustment network based on the three-layer feedback neural network. With the self-train of network, the parameters of the network are gained, which realizes the dual parameters adjustment of the flight height $h$ and Mach number Ma. Through simulation, control parameter optimization based on all characteristic points is ensured by the network. At the same time, the control parameters between all characteristic points is been grid, which makes flight control system stable even in the untrained characteristic points. Finally, each control parameter of flight and whole model of flight control system are computed out.
\end{abstract}

Keywords-Parameter adjustment, neural network, dual parameters, control rules, characteristic points

\section{INTRODUCTION}

With the advantages of self-study and train, the neural network is widely applied to the industry process, and it is extend to the flight control system. For example, navigation panel failure diagnosis of flight is imposed by neural network $^{[1]}$; self-adapted control rules of navigation panel failure is realized by online train feedback network ${ }^{[2]}$; flight parameters is estimated by the feedback network ${ }^{[3]}$; selfadapted flight control system with unknown parameters is realized online by the neural network ${ }^{[4 \sim 6]}$.

\section{DESIGN OF FLIGHT STABLE LOOP BASED ON}

\section{CHARACTERISTIC POINTS}

Flight control system is consist of measurement equipments (gesture angle gyroscope, velocity gyroscope and acceleration speedometer, etc), amplifier, controller and flight. Its framework is shown in the Figure 1.

Input variable is elevator deflecting angle, and state variable are pitching angle speed $\left(\omega_{z}\right)$, pitching angle $(\vartheta)$ and attacking angle $(\alpha)$, and output variable is ${ }^{\omega_{z}}$, which $\dot{\vartheta}=\omega_{z}, \theta_{\text {is }}$ the trajectory angle. The state space equation is shown as formula one.

$$
\begin{aligned}
& \left(\begin{array}{c}
\dot{\alpha} \\
\dot{\omega}_{z} \\
\dot{\vartheta}
\end{array}\right)=\left[\begin{array}{ccc}
-a_{4} & 1 & 0 \\
-a_{2} & -a_{1} & 0 \\
0 & 1 & 0
\end{array}\right]\left(\begin{array}{c}
\alpha \\
\omega_{z} \\
\vartheta
\end{array}\right)+\left(\begin{array}{c}
0 \\
-a_{3} \\
0
\end{array}\right) \delta_{z_{1}} \\
& y=\left(\begin{array}{lll}
0 & 1 & 0
\end{array}\right)\left(\begin{array}{c}
\alpha \\
\omega_{z} \\
\vartheta
\end{array}\right)=\omega_{z}
\end{aligned}
$$

Flight control system is designed by the method based on characteristic points. The time points of 0.35 second and 19.2 second are the most unstable in the whole flight control system, so the two time points is selected out for researching about the characteristic points. Then the four time points $(0.35 \mathrm{~s} 、 19.2 \mathrm{~s} 、 33.8 \mathrm{~s} 、 131.2 \mathrm{~s})$ are chose for the research object. Gas and power parameters of certain type of flights in the four characteristic points can be inquired from the table I.

TABLE I THE GAS AND POWER PARAMETERS
\begin{tabular}{|c|c|c|c|c|}
\hline $\mathrm{t}(\mathrm{s})$ & $a_{1}$ & $a_{2}$ & $a_{3}$ & $a_{4}$ \\
\hline 0.35 & 0.1029 & 0.0536 & 0.2553 & 2.9281 \\
\hline 19.2 & 3.376 & 23.39 & 37.62 & 0.02094 \\
\hline 33.8 & 34.87 & 29.34 & 39.12 & 0.000057 \\
\hline 131.2 & 37.01 & 44.92 & 42.72 & 0.00006 \\
\hline
\end{tabular}

The parameters from the table one is transformed into the formula one. Then the state space equation is shown as the formula 2 .

$$
\begin{aligned}
& A_{1}=\left[\begin{array}{cc}
-2.9281 & 1 \\
-0.0536 & -0.1029
\end{array}\right], B_{1}=\left[\begin{array}{c}
0 \\
-0.2553
\end{array}\right], C_{1}=\left[\begin{array}{ll}
0 & 1
\end{array}\right] \\
& A_{2}=\left[\begin{array}{cc}
-0.02094 & 1 \\
-23.39 & -3.376
\end{array}\right], B_{2}=\left[\begin{array}{c}
0 \\
-37.62
\end{array}\right], C_{2}=\left[\begin{array}{ll}
0 & 1
\end{array}\right] \\
& A_{3}=\left[\begin{array}{cc}
-0.000057 & 1 \\
-29.34 & -34.87
\end{array}\right], B_{3}=\left[\begin{array}{c}
0 \\
-39.12
\end{array}\right], C_{3}=\left[\begin{array}{ll}
0 & 1
\end{array}\right] \\
& A_{4}=\left[\begin{array}{cc}
-0.00006 & 1 \\
-44.92 & -37.01
\end{array}\right], B_{4}=\left[\begin{array}{c}
0 \\
-42.72
\end{array}\right], C_{4}=\left[\begin{array}{ll}
0 & 1
\end{array}\right]
\end{aligned}
$$

in the middle, then

$$
\begin{gathered}
G_{g}(s)=\frac{K_{g}}{T_{g}^{2} s^{2}+2 T_{g} \zeta_{g} s+1} \\
K_{g}=0.056, T_{g}=0.01, \zeta_{g}=0.6 \\
G_{c 1}(s)=\frac{1+0.02 s}{1+0.5 s} \quad K_{p}\left(1+\frac{1}{T_{I} s}+T_{D} s\right)
\end{gathered}
$$


Transfering function of the loop system is shown in the formula 3 .

$$
G(s)=K_{p} \frac{T_{I} T_{D} s^{2}+T_{I} s+1}{T_{P} s} \frac{K_{w}\left(T_{1} s+1\right)}{T_{c}^{2} s^{2}+2 \zeta_{c} T_{c} s+1} \cdot \frac{\dot{K_{\delta}}}{T_{\delta}^{2} s^{2}+2 \zeta_{\delta} T_{\delta} s+1} \cdot \frac{K_{g}}{T_{g}^{2} s^{2}+2 \zeta_{g} T_{g} s+1}
$$

Then

$$
\begin{gathered}
T_{I} T_{D} s^{2}+T_{I} s+1=\left(T_{c}^{2}+T_{\delta}^{2}+2 \zeta_{c} T_{c} \times 2 \zeta_{\delta} T_{\delta}^{\prime}\right) s^{2}+\left(2 \zeta_{\delta} T_{\delta}^{\prime} \times 2 \zeta_{c} T_{c}\right) s+1 \\
\left\{\begin{array}{c}
T_{I} T_{D}=T_{c}^{2}+T_{\delta}^{\prime 2}+2 \zeta_{c} T_{c} \times 2 \zeta_{\delta}^{\prime} T_{\delta}^{\prime} \\
T_{I}=2 \zeta_{\delta}^{\prime} T_{\delta}^{\prime} \times 2 \zeta_{c} T_{c} \\
2 \zeta_{\delta}^{\prime} T_{\delta}^{\prime}=0.02,
\end{array} T_{\delta}^{2}=0.0002\right.
\end{gathered}
$$

Then parameters of $T_{I}$ and $T_{D}$ is calculated out shown as the table II.

\begin{tabular}{|c|c|c|c|}
\multicolumn{5}{|c}{ TABLE II.THE PARAMETERS OF PID } \\
\hline $\mathrm{t}(\mathrm{s})$ & $K_{p}$ & $T_{I}$ & $T_{D}$ \\
\hline 0.35 & 5 & 8.56 & 0.3491 \\
\hline 19.2 & 0.6 & 0.593 & 0.7299 \\
\hline 33.8 & 0.65 & 0.53 & 0.66 \\
\hline 131.2 & 0.8 & 0.44 & 0.514 \\
\hline
\end{tabular}

The result of flight stable control loop by simulation software named simulink is shown on Fig 2.

\section{SELF-STUDY AND TRAIN OF PARAMETER ADJUSTMENT}

\section{OF NN}

In case of the three-layer feedback network, the parameters of control network are trained by the four characteristic points. The hided layer in the network has 6 nodes. The input layer of network has 2 nodes which are respectively corresponding to the flight height $h$ and Mach number $M a$. The output layer of network has 3 nodes which are respectively corresponding to the three parameters of PID: $K_{p} 、 T_{i} 、 T_{d}$, which is shown in the Fig 3.

The input of network is $x=\left(x_{1}, x_{2}\right)^{T}=(h, M a)^{T}$.

The output of network is $y=\left(y_{1}, y_{2}, y_{3}\right)^{T}=\left(K_{p}, T_{i}, T_{d}\right)^{T}$, $(x(k), y(k)), k=1, \cdots, 4$.

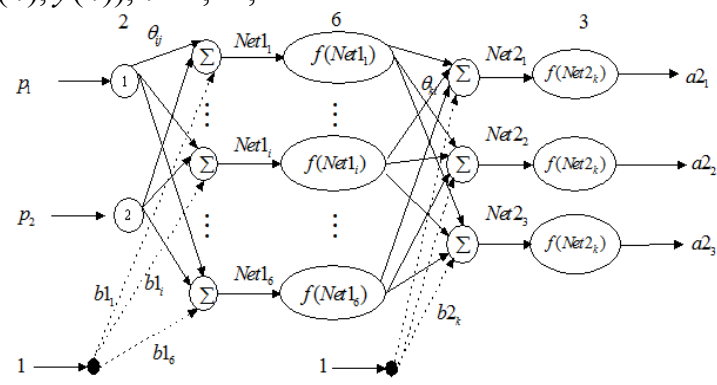

Figure 3. The framework of parameter adjustment of NN

\section{RESULT ANALYSIS OF SIMULATION ABOUT}

\section{PARAMETER ADJUSTMENT OF NN}

The parameters of PID in the uncharacteristic points of control system based on the parameter adjustment of neural

\begin{tabular}{|c|c|c|c|}
\hline$t(s)$ & $K_{p}$ & $T_{i}$ & $T_{d}$ \\
\hline 0.35 & 5 & 8.56 & 0.3491 \\
\hline 1.04 & 4. 68 & 6.2 & 0.54 \\
\hline 2.74 & 3.12 & 4. 284 & 0.684 \\
\hline 3.8 & 1.9 & 5. 14 & 0.31 \\
\hline 4. 4 & 1. 2 & 4. 2 & 0.34 \\
\hline 7.7 & 2.2 & 4. 18 & 0.24 \\
\hline 14.8 & 1.1 & 4.07 & 0.54 \\
\hline 19.2 & 0.6 & 0.593 & 0.7299 \\
\hline 21 & 0.668 & 0.500 & 0.6400 \\
\hline 33.8 & 0.65 & 0.53 & 0.66 \\
\hline 47.4 & 0.6485 & 0.477 & 0.6483 \\
\hline 56 & 0.8418 & 0.702 & 0.5659 \\
\hline 83 & 0.7031 & 0.540 & 0.6250 \\
\hline 131. 2 & 0.8 & 0.44 & 0.514 \\
\hline 164 & 0.7820 & 0.632 & 0.5914 \\
\hline
\end{tabular}
network above is shown in the table 3 . The result of simulation of flight control system is shown from the Fig 4 to the Fig 13.

From the Fig 4 to Fig 13, the stable output is ensured by the parameter adjustment of neural network. So all controlling parameters is designed to be optimal relatively. At the same time, the output of the control system is stable. 


\section{CONCLUSIONS}

In the paper, there are some characteristic points selected from the flight line, and small disturbance linear equations of flight are gained through small deviation linearization aircraft motion equation. Then a set of flight models is gotten with the equations. According to the controlled object the establishment of a complete vehicle control system stable loop; Then design a kind of three layers of BP (Back Propagation neural network parameters tuning device, it can replace the traditional according to dynamic pressure, height or a single parameters tuning ware, Mach number by using the neural network parameters tuning of flight control law is to gain parameters tuning are given, finally the whole flight packages line of control law. Aircraft in flight process, system dynamic to find this control law form, scheduling flying control law to achieve the stability of the whole flight packages the purpose of flight online.

\section{REFERENCES}

[1] Liang Di, Su Zhixun. Orthogonal Picture Based Human Face Reconstruction Technology, Journal of System Simulation, 2003, Vol. 15 No. 11: 1646 Rysdyk R. T., Calise A. J.. Fault tolerant flight control via adaptive neural network augmentation. AIAA guidance and control conference (98-4483), USA: 1998.

[2] Totah J.. Adaptive flight control and on2line learning. AIAA guidance and control conference (97-537), USA: 1997. 78-83.

[3] Jing Zhou, Xinhan Huang. Application of a nonlinear fault detection and isolation scheme to an aerocraft's nonlinear closed-loop system, Information Technology Journal. 2008, v 7, n 8, pp. 1163-1169.

[4] Jing Zhou, Xinhan Huang. Application of a new Fault Detection Approach to Aerocraft's Closed-loop Control System, Lecture Notes in Computer Science. 2008.1223-1232.

[5] Nho K., Agarwal R.. Neural network model2based predictive control of aircraft dynamics. AIAA guidance and control conference(20000891),USA: 2000. 102-109.

[6] Li Gaicong, Liu Yiguang, You Zhisheng. A 3D Reconstruction Method for Car-body Surface in Binocular, Journal of Sichuan Normal University(Natural Science), 2002, Vol. 25 No. 3: 320 323.

[7] Swith D. W., Ward D. T., Nguyen T. X.. An improved artificial neural network flight mode interpreter. AIAA guidance and control conference (98-4201), USA: 1998. 75-82.

[8] Jing Zhou, Wei Guo. A new LMI-based Filter Scheme for Fault Detection. Advances In Systems Science and Applications. 2009, v 9, n 1, pp. 158-165.

[9] Jianqing Zhang, Jun Tao, Zuxun Zhang. A flexible technique for the slide projector calibration, Proceedings of SPIE - The International Society for Optical Engineering, Beijing, China, 2003, v 5286, n 1, pp. 187-190.

[10] Jun Tao, Jianqing Zhang, Zuxun Zhang. Calibration of a Projector with a Planar Grid, ISPRS, Istanbul, Turkey, 2004.

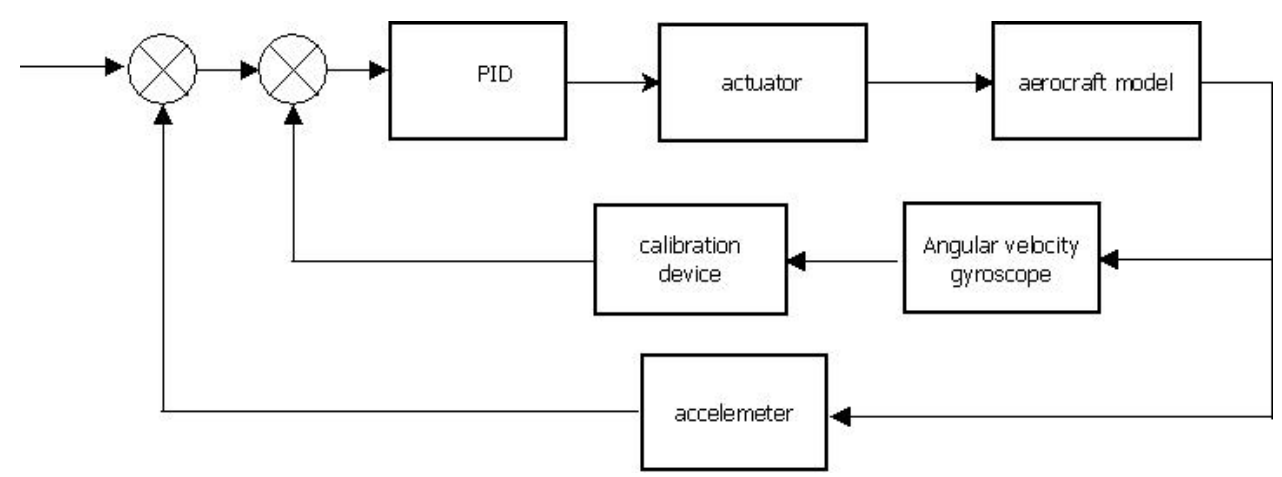

Figure 1. The framework of flight loop control system

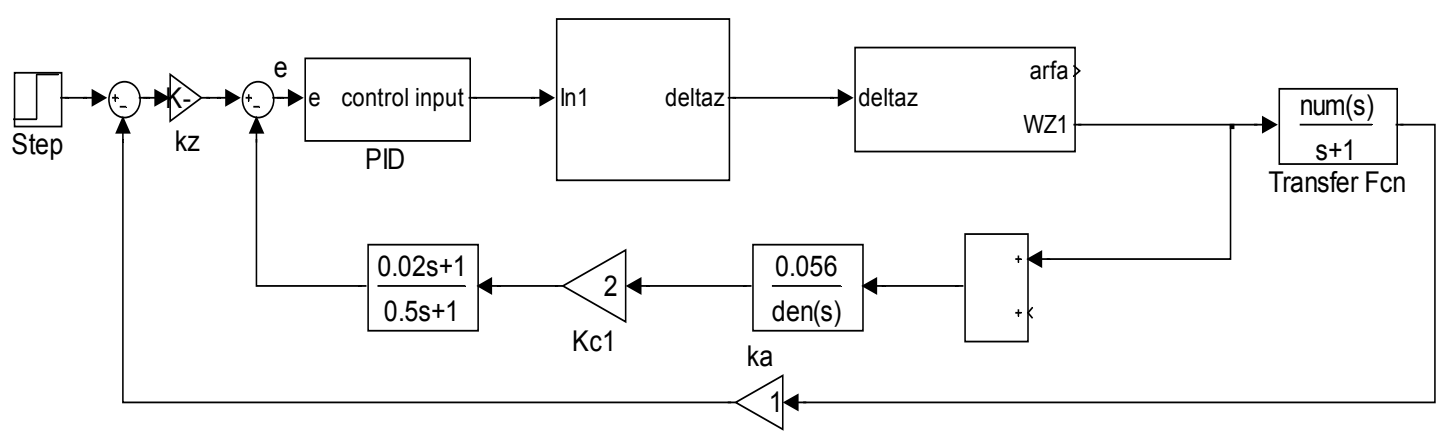

Figure2. The result of realtime simulation 

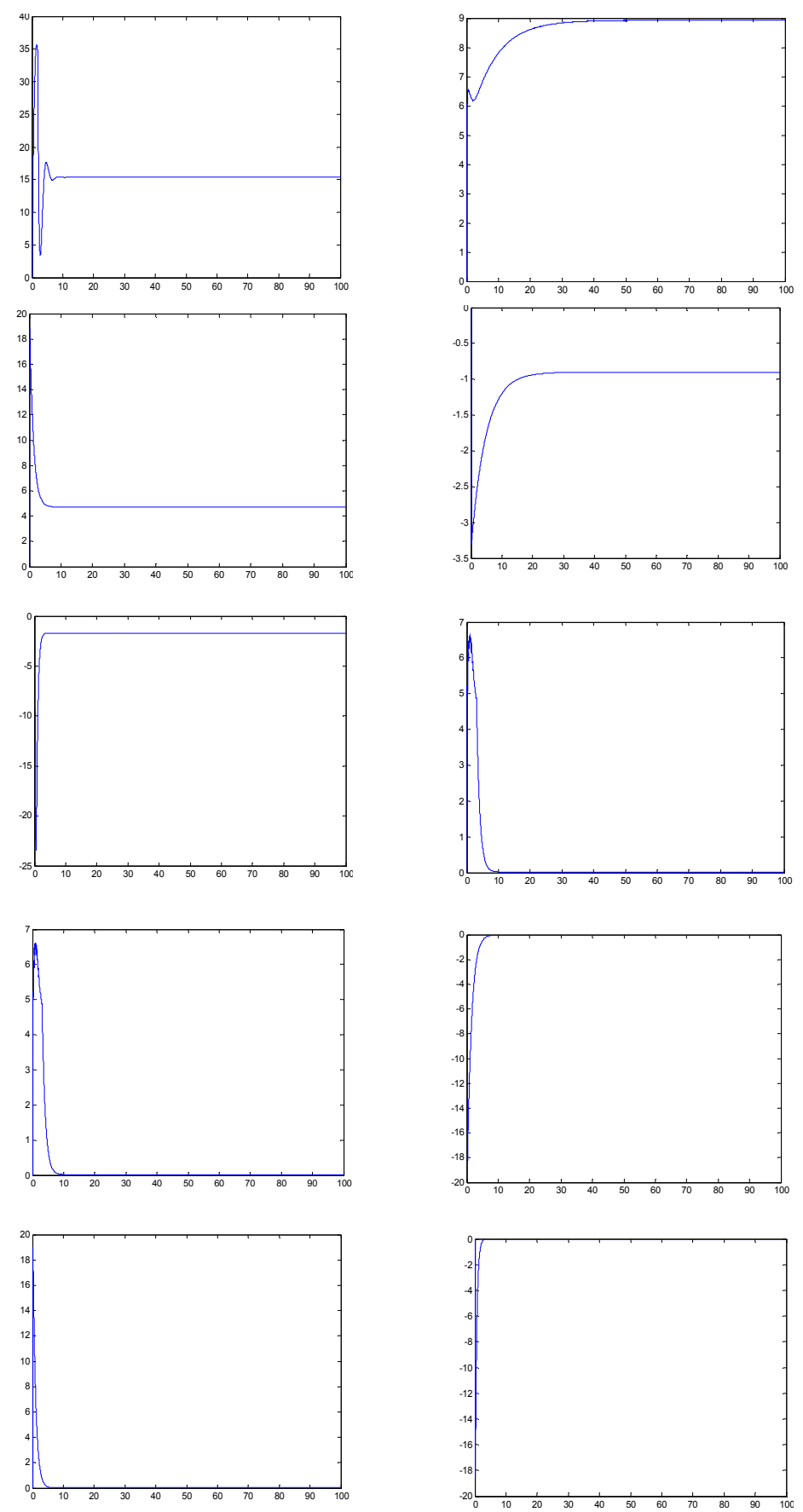

Figure 4 to Figure 13 the result of simulation 